\title{
CRESCIMENTO INICIAL DE AÇAÍZEIRO EM SISTEMA AGROFLORESTAL NO P. A. BELO HORIZONTE I, SÃO DOMINGOS DO ARAGUAIA, PARÁ
}

\author{
Tatiane Pereira Guimarães ${ }^{1}$; Rosana Quaresma Maneschy ${ }^{2}$; Andréa Hentz de Mello ${ }^{3}$; Albinei \\ Araujo de Castro ${ }^{4}$; Ilmaione Keiza de Souza Oliveira ${ }^{5}$; Karolinny Carneiro Guerra Costa ${ }^{6}$ \\ ${ }^{1}$ Discente de Agronomia, Faculdade de Ciências Agrárias de Marabá (FCAM), Universidade Federal do Pará (UFPA), \\ Bolsista CNPq, tatianepg.ufpa@gmail.com \\ ${ }^{2}$ Prof. a, DSc., FCAM, UFPA, romaneschy@ufpa.br \\ ${ }^{3}$ Prof. a, DSc., FCAM, UFPA, andreahentz@ufpa.br \\ ${ }^{4}$ Discente de Agronomia da UFPA - Campus de Marabá, Bolsista FAPESPA, albinei_araujo@hotmail.com. \\ ${ }^{5}$ Discente de Agronomia da UFPA - Campus de Marabá, , ilmaionekeiza@ hotmail.com. \\ ${ }^{6}$ Discente de Agronomia da UFPA - Campus de Marabá, Bolsista PIBIC/PARD, karolinnycg.guerra@gmail.com.
}

\begin{abstract}
RESUMO: Os sistemas agroflorestais (SAF) são utilizados por agricultores familiares para recuperação de áreas degradadas e diversificação do sistema de produção. Objetivou-se avaliar o crescimento inicial do açaizeiro (Euterpe oleracea Mart.) em um SAF implantado por agricultores familiares a fim de recuperar a área de mata ciliar da unidade de produção, no assentamento rural Belo Horizonte I, localizado no município de São Domingos do Araguaia-PA. Os parâmetros utilizados para a avaliação foram: a taxa de sobrevivência, altura total da planta $(\mathrm{m})$ e diâmetro do coleto (mm). A primeira avaliação ocorreu aos 45 dias após o plantio das mudas e as seguintes a cada 30 dias. Apesar das condições adversas de implantação e consequente baixa taxa de sobrevivência, considerou-se satisfatório o crescimento dos indivíduos que sobreviveram no período avaliado.
\end{abstract}

PALAVRAS-CHAVE: agricultura familiar, biodiversidade, Euterpe oleracea.

\section{AÇAÍ GROWTH IN THE AGROFORESTRY SYSTEM IN P. A. BELO HORIZONTE I, SÃO DOMINGOS DO ARAGUAIA, PARÁ}

\begin{abstract}
Agroforestry system (AFS) are used by smallholders for rehabilitation of degraded areas and diversification of production systems. The objective was to evaluate the initial growth of açaí (Euterpe oleracea Mart.) implanted in a AFS by family farmers in order to recover the riparian area of the plant. In rural settlement Belo Horizonte I, located in São Domingos do Araguaia-PA. The parameters used for evaluation were: survival rate, total plant height $(\mathrm{m})$ and stem diameter $(\mathrm{mm})$. The first evaluation at 45 days after planting the seedlings and the following every 30 days. Despite the adverse conditions of implantation and consequent low rate of survival was considered satisfactory growth of individuals who survived the study period.
\end{abstract}

KEY-WORDS: biodiversity, family farm, Euterpe oleracea.

\section{INTRODUÇÃO}

A questão ambiental tem sido foco de atenção da comunidade científica de diferentes países, subsidiando a formulação de políticas que permitam conciliar a produção com a satisfação de necessidades humanas e a conservação e uso racional dos recursos naturais, sendo a agricultura de grande escala uma das principais responsáveis pela degradação ambiental por meio de 
desmatamentos, empobrecimento do solo, contaminação de recursos hídricos dentre outros impactos insustentáveis (MACIEL, 2009).

Durante séculos, o homem vem interferindo na floresta, explorando de forma predatória a madeira e seus produtos não madeireiros. Outras formas de uso da terra como a pecuária extensiva, a agricultura de corte e queima, e mais recentemente a agricultura mecanizada vem contribuindo para o desmatamento da Amazônia. Estima-se que nos últimos 10 anos, cerca de 200 mil km² foram desmatadas (ALMEIDA; SABOGAL; BRIENZA-JUNIOR, 2006).

Neste contexto, para mitigar os impactos da degradação das pastagens. os sistemas agroflorestais (SAF) têm sido considerados alternativa de uso da terra menos impactante ao meio ambiente, sendo inclusive indicado para recuperação de áreas de proteção permanente e reserva legal. Esses sistemas proporcionam subsistência aos agricultores, em geral são economicamente viáveis além de cumprir várias funções ecológicas (FENDEL, 2007).

Os SAF integram cultivos temporários, às essências florestais e/ou fruteiras, também podendo estar associado às pastagens e animais (DUBOIS et al., 1996). Objetivou-se avaliar o crescimento inicial do açaizeiro (Euterpe oleracea Mart.) em um sistema agroflorestal implantado por agricultores familiares, a fim de recuperar a área de mata ciliar da unidade de produção.

\section{MATERIAL E MÉTODOS}

A pesquisa foi realizada no âmbito do projeto "Sistemas silvipastoris e agrossilvipastoris como alternativa para a sustentabilidade da pecuária na agricultura familiar da região de Marabá - PA" desenvolvido pela Universidade Federal do Pará - Campus de Marabá com apoio financeiro da Fundação de Amparo à Pesquisa do Estado do Pará (FAPESPA). O local da pesquisa foi no P. A. Belo Horizonte I, situado à altura do km 30 da BR-153, no Município de São Domingos do Araguaia, na Microrregião de Marabá. O clima local é de Afi no limite de transição para Awi com temperatura média de $28,0{ }^{\circ} \mathrm{C}$ (ALMEIDA, 2007). Solo podzólico vermelho amarelo com textura argilo-arenosa (NAVEGANTESALVES, 2009).

Foi implantada, em fevereiro de 2010, uma ação-teste agroflorestal em lote de agricultor familiar. O local da ação-teste, as espécies e o desenho do SAF foram determinados pela família, caracterizando a abordagem participativa e de construção de inovações da pesquisa (CHIA et al., 2006).

As mudas de abacate (Persea americana L), ipê amarelo (Tabebuia serratifolia Valh) e ipê branco (Tabebuia roseoalba Ridl.) foram produzidas em um viveiro comunitário no assentamento, sendo que as de açaí foram doadas pelo Sindicato dos Agricultores de São Domingos do Araguaia e as de nim (Azadirachta indica A. Juss) pela EMATER. 
A área escolhida, de 0,25 ha, era inicialmente de floresta primária, que foi suprimida há aproximadamente 15 anos para formação de pastagem com braquiarão (Brachiaria brizantha cv. marandu Hochst. ex A. Rich). As pastagens foram utilizadas por 10 anos e nos últimos cinco anos estão em pousio.

O solo em que foi implantada a açãoteste tem as seguintes características: Areia grossa $=174 \mathrm{~g} \mathrm{~kg}^{-1}$; Areia fina $=529 \mathrm{~g} \mathrm{~kg}^{-1}$; Silte $=197 \mathrm{~g} \mathrm{~kg}^{-1} ; \mathrm{pH}=5,6 ; \mathrm{P}=5 \mathrm{mg} \mathrm{dm}^{-3} ; \mathrm{K}$ $=77 \mathrm{mg} \mathrm{dm}^{-3} ; \mathrm{Na}=27 \mathrm{mg} \mathrm{dm}{ }^{-3} ; \mathrm{Ca}=2,8$

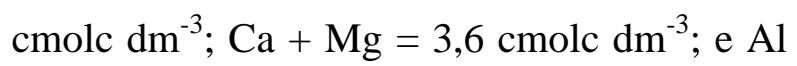
$=0,1 \mathrm{cmolc} \mathrm{dm}^{-3}$.

O preparo da área foi de roçagem manual, a implantação das mudas se deu de forma aleatória (não configurando linhas de plantio específicas por espécie) e o espaçamento utilizado foi de $3 \mathrm{~m} \times 2 \mathrm{~m}$.

Para a avaliação da espécie frutífera foi considerado o efeito de bordadura e, portanto não foram avaliadas as fileiras externas de plantas do SAF. Os parâmetros utilizados para a avaliação foram: a taxa de sobrevivência, altura total da planta (m) e diâmetro do coleto ( $\mathrm{mm}$ ).

A primeira avaliação ocorreu aos 45 dias após o plantio das mudas e as seguintes a cada 30 dias até maio de 2011. Na ocasião das avaliações também eram realizadas entrevistas não diretivas com os membros da família responsáveis pela ação-teste.

Foram testadas equações de regressão para estimar os padrões de crescimento avaliados em relação à idade do plantio. A acurácia dos modelos de regressão foi avaliada por meio do teste $\mathrm{F}$ ao nível de $5 \%$.

\section{RESULTADOS E DISCUSSÃO}

A escolha do açaí para compor a açãoteste agroflorestal se deu porque na região é uma espécie naturalmente encontrada em matas ciliares e tem potencial econômico. A ação-teste foi implantada em uma capoeira de cinco anos somente no final do período chuvoso, pois era o momento em que a família tinha disponibilidade de mão-de-obra.

As mudas de açaí foram plantadas possuindo apenas três folhas, mas segundo a Comissão Estadual de Sementes e Mudas do Pará (1997) o recomendado é ter cinco folhas. Esses fatores podem justificar a baixa taxa de sobrevivência do açaí (Figura 1), sobretudo após o período seco do ano, pois se trata de uma espécie exigente a água. Segundo Calzavara (1972) o cultivo do açaizeiro é indicado para as condições tropicais de grande precipitação pluviométrica e elevada temperatura.

As relações dos parâmetros de crescimento nos períodos avaliados na açãoteste agroflorestal foram consideradas significativas $(\mathrm{P} \geq 0,05)$ (Figura 2).

Foi observado que o único trato cultural realizado na ação-teste durante o período de avaliação foi a capina com a finalidade de diminuir a competição entre as espécies introduzidas e a gramínea. Apesar de ter sido realizada análise de solo, não foi feito 
nenhum tipo de correção ou adubação dessa área. A análise evidenciou que o solo de várzea apresentou $\mathrm{o} \quad \mathrm{pH}$ ácido. $\mathrm{A}$ recomendação para o açaí de terra firme no primeiro ano de plantio seria a aplicação de 45 g/planta de N, 60 g/planta de P, 40 g/planta de $\mathrm{K}$, tendo em vista que de acordo com a análise da área estudada os níveis de teores nutricionais encontrados podem ser considerados médios (OLIVEIRA et al, 2002). Os estudos sobre a recomendação de adubação e correção do solo em áreas de açaí de várzea ainda são muito incipientes, ficando difícil estabelecer recomendações mais especificas para os indivíduos desse estudo de caso.

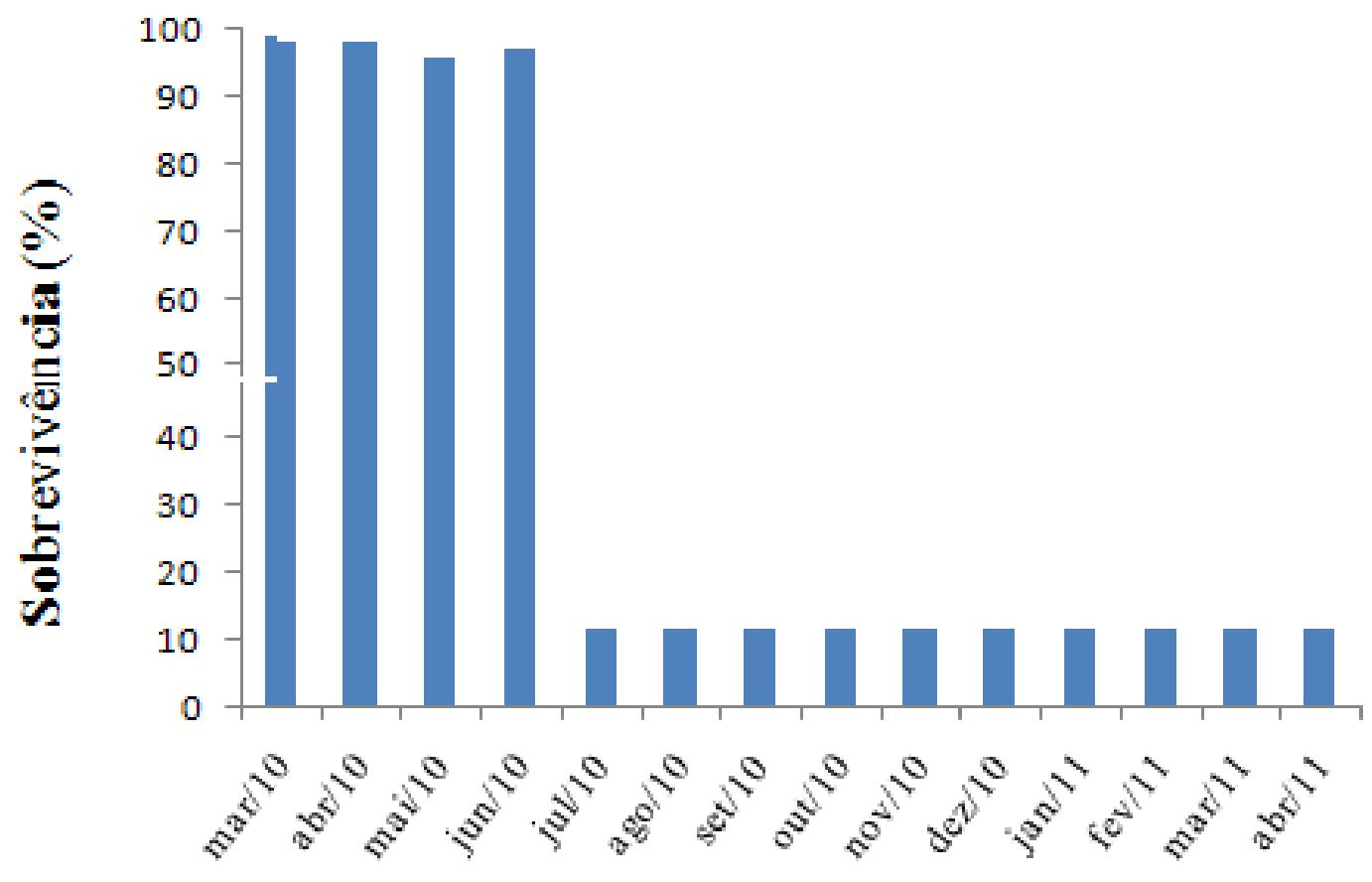

Figura 1. Taxa de sobrevivência do açaí em ação-teste agroflorestal de unidade de produção familiar no P. A. Belo Horizonte I, São Domingos do Araguaia, Pará. 

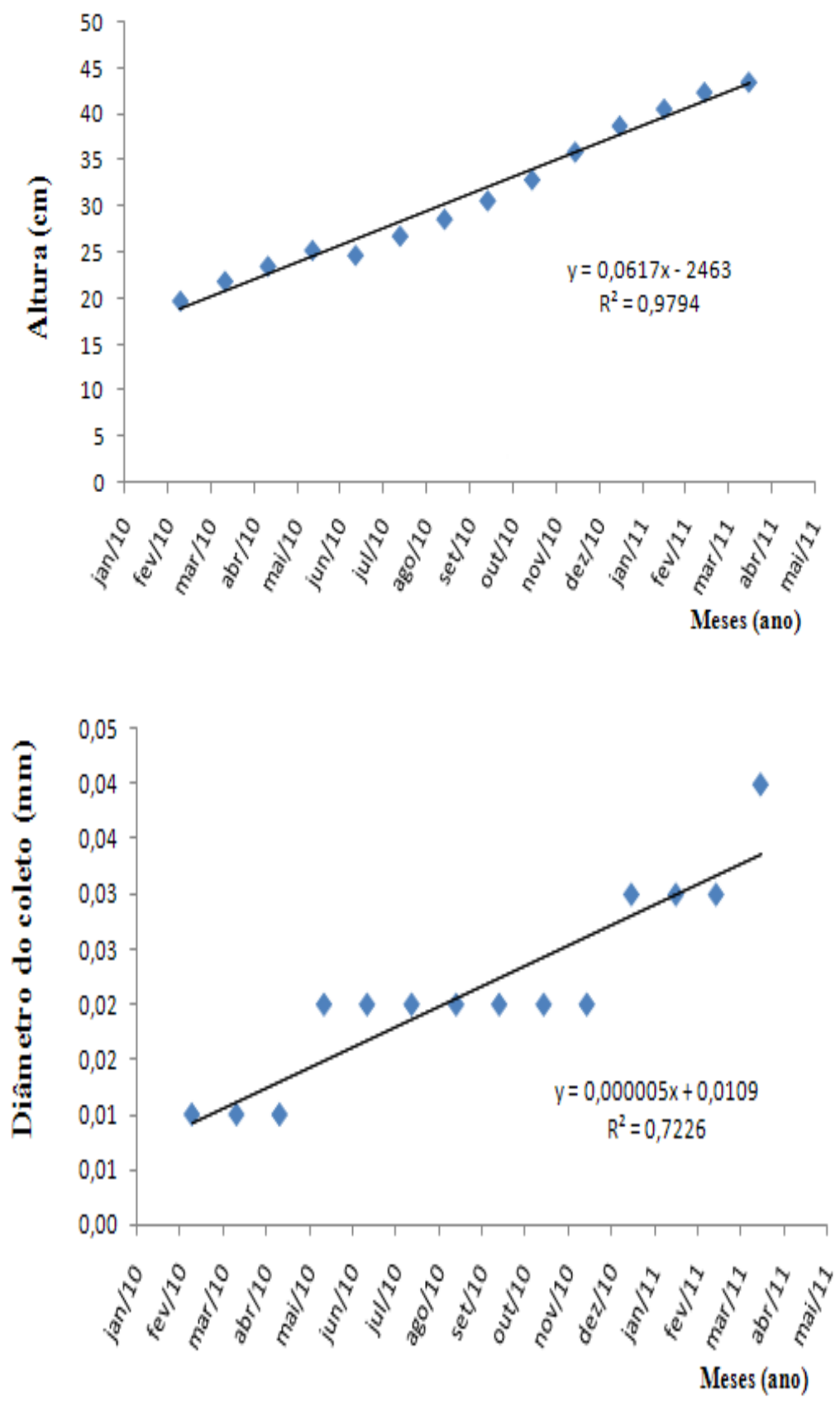

Figura 2. Relações dos parâmetros de altura total e diâmetro do coleto do açaí com a idade de plantio em ação-teste agroflorestal de unidade de produção familiar no P. A. Belo Horizonte I, São Domingos do Araguaia, Pará.

Nesse contexto de agricultura familiar o recomendado mesmo seria a utilização de adubos orgânicos como: estercos bovinos/suínos/aves, húmus, vermicompostagem, ou ainda a adubação verde, que será utilizada, pois em março de 2011 a família introduziu estacas de gliricídia por toda a área do SAF. As práticas aplicadas pelos agricultores na ação teste foram conduzidas de acordo com a realidade e 
disponibilidade da família para o desenvolvimento da ação.

Entre as restrições enfrentadas para o estabelecimento do açaí a campo pode-se relacionar: estádio de crescimento das mudas, momento inadequado do plantio das mesmas, falta de adubação (química e/ou orgânica) e disponibilidade de mão de obra para realização das atividades exigidas (fabricação de composto orgânico, tratos culturais, limpeza e irrigação).

\section{CONCLUSÕES}

O açaí apresentou baixa taxa de sobrevivência nas condições estudadas. Apesar das condições adversas de implantação do SAF considerou-se satisfatório o crescimento dos indivíduos que sobreviveram no período avaliado.

\section{REFERENCIAS}

ALMEIDA, E.; SABOGAL, C.; BRIENZA JÚNIOR, S. Recuperação de Áreas Alteradas na Amazônia Brasileira: Experiências locais, lições aprendidas e implicações para políticas públicas. $1 \mathrm{ed}$. Belém, Brasil: CIFOR e Embrapa Amazônia Oriental, 2006. 202 p.

ALMEIDA, M. F. Caracterização Agrometeorológica do Município de Marabá/PA. 2007. 77f. Trabalho de Conclusão de Curso (Agronomia) Faculdade de Ciências Agrárias de Marabá, Universidade Federal do Pará, Marabá, 2007.
CALZAVARA, B. B. G. As possibilidades do açaizeiro no Estuário Amazônico. Belém: Boletim da Faculdade de Ciências Agrárias do Pará. 1972. 103 p.

CHIA, E.; DUGUÉ, P.; SAKHO-JIMBIRA, S. Les exploitations agricoles familiales sontelles des institutions? Agricultures, v. 15, n. 6, p. 498-505, 2006.

COMISSÃO ESTADUAL DE SEMENTES E MUDAS DO PARÁ. Belém, Pará. Normas técnicas e padrões para a produção de mudas fiscalizadas no Estado do Pará. Belém, 1997. 40p.

DUBOIS, J. C. L.; VIANA, V. M.; ANDERSON, A. B. Manual Agroflorestal para a Amazônia. Vol. 1. Rio de Janeiro, Brasil: REBRAF. 1996. 228 p.

FENDEL K. L. Recuperação de Mata Ciliar com Sistema Agroflorestal. 2007. 70 f. Trabalho de Conclusão (Ciências Biológicas) - Centro de Ciências Tecnológicas, Universidade do Vale do Itajaí, Itajaí, 2007.

MACIEL, V. R. Implantação de Um Sistema Agroflorestal Como Forma de Recuperação de Uma Área Degradada. São Paulo. Rev. Bras. De Agroecologia/nov. 2009 Vol. 4 No. 2. 2009. 
NAVEGANTES-ALVES, L. Gestion des pâturages en Amazonie Orientale: les décisions des pratiques. Montpellier, França: SUPAGRO/SIBAGHE, 2009 - Tese de doutorado em andamento. 2009.
OLIVEIRA, M. S. P.; CARVALHO J. E. U.; NASCIMENTO, W. M. O.; MÜLLER, C. H. Cultivo do Açaizeiro para Produção de Frutos. Belém: Embrapa Amazônia Oriental, 2002. 18 p. 